\title{
Are BAX and JNK involved in inhibition of placental growth in rats with dexamethasone-induced intrauterine growth restriction
}

\begin{abstract}
Molecular mechanisms involved in the onset and progression of intrauterine growth restriction (IUGR) are not clear, although enhanced apoptosis seems to play a primary role. The present study investigated two key proteins, B-cell lymphoma associated X protein and c-Jun N-terminal kinase, in the basal and labyrinth zones of Dexamethasone-induced IUGR placenta. Pregnant Sprague-Dawley rats received daily intra-peritoneal injections of either dexamethasone or saline starting from 14 days of gestation to 21. Dams were sacrificed on day 19 and 21; fetuses and placentas were collected. IUGR was seen at day 21 with significantly reduced fetal weights $(\mathrm{p}<0.05)$. Gene and protein expressions of B-cell lymphoma associated $\mathrm{X}$ protein and c-Jun $\mathrm{N}$-terminal kinase in the placental basal and labyrinth zones were investigated by real-time polymerase chain reaction, western blotting, and immune histochemistry. Expression of both B-cell lymphoma associated X protein and c-Jun N-terminal kinase mRNA decreased in the basal zone and increased in the labyrinth zone $(p<0.05)$ with no significant change detected at the protein level. The increase in expression of both B-cell lymphoma associated X protein and c-Jun N-terminal kinase in the labyrinth zone may indicate that both signaling molecules have a significant role in the apoptosis of this zone.
\end{abstract}

Volume 5 Issue 3 - 2020

\author{
Amr Osman, ${ }^{1,2}$ Maie D Al Bader, ${ }^{2}$ Mariam \\ Alqaryyan,, Majeda Al Zuabi,' Nada Ayed,' \\ Modhi Khaled,' Shaikhah Al Houli,' Narayana \\ Kilarkaje ${ }^{3}$ \\ 'Ministry of Health, Kuwait \\ ${ }^{2}$ Department of Physiology, Kuwait University, Kuwait \\ ${ }^{3}$ Department of Anatomy, Kuwait University, Kuwait
}

Correspondence: Amr Osman, Department of Physiology, Faculty of Medicine, Kuwait University, Salmiya, Hawally, Kuwait, Tel 0096599986785,Email amrosman00@gmail.com

Received: August 2I, 2020 | Published:September 30, 2020

Keywords: BAX, JNK, placenta, dexamethasone, intrauterine growth restriction

Abbreviations: ST, syncytiotrophoblast cells; BZ, basal zone; TG, trophoblast gaint cells; S, spongiotrophoblast cells; GC, glycogen cells; DC, deciduas cells; LZ, labyrinth zone

\section{Introduction}

Rat and human placentas are known as hemochorial placentas sharing many similarities. ${ }^{1,2}$ Hemochorial placentas are considered to be the most invasive type of placentas, in which erosion of all maternal tissue layers allow for a direct connection between the maternal and fetal blood. ${ }^{3}$ Trophoblast cells are considered to be the parenchymal cells of the hemochorial placenta. ${ }^{2}$ In rats, the placenta is divided into two main zones, the basal zone (BZ) and labyrinth zone (LZ). The BZ consists of three types of differentiated cells: spongiotrophoblast cells (S), trophoblast giant cells (TG), and glycogen cells (GC). ${ }^{3}$ This zone is important in placental steroid production at the end of gestation. ${ }^{4}$ The LZ, responsible for nutrient and waste transfer between mother and fetus. It is made up of three layers of trophoblasts: outer trophectoderm and two layers of syncytiotrophoblasts (ST); they function as placental barriers. ${ }^{3}$

In rats, dexamethasone (DEX) has been used to induce intrauterine growth restriction (IUGR)..$^{5-7}$ IUGR is a condition in which the fetal weight is at or lower than the $10^{\text {th }}$ percentile of the expected normal birth weight for the same gestational age or less than two standard deviations of the average weight of the fetuses. ${ }^{8} \mathrm{DEX}$ is known to affect normal placental development by altering placental trophic function, altering trophoblast development ${ }^{10}$ and increasing trophoblast apoptosis. ${ }^{11}$ This method of treatment to induce IUGR provides a relevant model for placental experiments since glucocorticoids are used in the treatment of mothers at risk of preterm delivery ${ }^{12}$ and those who are affected by autoimmune disorders that need to be controlled by steroid therapy.

A healthy rat placenta normally experiences apoptosis that is time and zone dependent. ${ }^{7}$ In IUGR placentas, apoptosis rate is higher than in normal placentas towards the end of gestation. ${ }^{8}$ Apoptosis is an energy dependent process with two main pathways: intrinsic, also known as the mitochondrial pathway, and extrinsic, also known as the death receptor pathway. ${ }^{13}$ Both pathways culminate at an execution pathway mediated by caspase- 3 and involve DNA fragmentation and eventual phagocytosis. ${ }^{13}$ The Bcl-2 family is a critical checkpoint of the intrinsic pathway. ${ }^{14}$ The Bcl-2 family includes pro-apoptotic and anti-apoptotic members, with B-cell lymphoma associated X protein (BAX) being an important pro-apoptotic member. ${ }^{15}$ BAX protein induces the release of cytochrome-C from mitochondria. ${ }^{16}$ The ratio of Bcl-2/BAX is important in setting the susceptibility threshold of the intrinsic pathway of apoptosis. BAX inserts into mitochondrial outer membrane in the form of homo-oligomerized multimers when a signal is received from caspase- $8 .{ }^{14}$ In the mitochondrial outer membrane and liposomes, oligomerized BAX is able to form supramolecular openings, which is involved in the release of cytochorme-C from the mitochondria. ${ }^{17}$ Another Bcl-2 family member that is invovled in in the intrinsic pathway of apoptosis is Bcl-2 homologous antagonist killer (BAK). Following apoptotic activation signaling, BAK translocates from cytosol to mitochondria to release cytochrome C..$^{18,19}$

C-Jun N-terminal protein kinases (JNK), which is also known as stress-activated protein kinase, is a protein that can affect gene transcription via phosphorylation of transcription factors. ${ }^{20,21} \mathrm{JNKs}$ are a part of the mitogen activated protein kinases [MAPK] family that respond to different stress signals and play a role in the intrinsic 
and extrinsic pathways of apoptosis. ${ }^{22} \mathrm{JNKs}$ induce apoptosis by transactivating certain transcription factors, therefore, upregulating the pro-apoptotic genes, or through acting on mitochondrial proand anti-apoptotic proteins by modulating their activity via specific phosphorylation events. ${ }^{23}$ Differences in JNK signal intensity and duration lead to different outcomes with short-term activation promoting survival and prolonged activation inducing apoptosis. ${ }^{24}$ JNK can mediate apoptosis directly through affecting the transcription of apoptotic genes and/or through a transcriptional-independent mechanism; also it can phosphorylate pro- and anti-apoptotic proteins and can prevent the ubiquitination and degradation of its substrates through phosphorylation. It is additionally involved in increasing the stability of p53 along with increasing its transcriptional activity and enhancing its apoptotic effect. ${ }^{21,25} \mathrm{JNK}$ has a role in regulating BAX activity but it is not completely understood. It was shown that JNK can promote Bax translocation to mitochondria in stress-induced apoptosis through phosphorylation. ${ }^{26,27}$ This can be achieved through phosphorylation of 14-3-3, a cytoplasmic anchor of Bax, which lead to dissociation of BAX from the anchor and allowing translocation to the nucleus. ${ }^{27}$

In this experiment, DEX will be used to induce IUGR. We aim to explore the expression of BAX and JNK on the gene level by PCR and their translation into proteins through western blotting and localization within the cell using immune histochemistry.

\section{Materials and methods}

\section{Animal, experimental design, tissue preparation, and collection}

Adult female Sprague-Dawley rats (280-300g) were kept in the Animal House at the Health Sciences Centre of Kuwait University and guidelines of the Animal House were followed. The rats were provided with food and water ad libitum and were kept on a 12:12 cycle of lightness and darkness in a room temperature between 20 $22^{\circ} \mathrm{C}$. For mating, adult male rats $(280-300 \mathrm{~g})$ were placed with two females. The day of positive vaginal smear was considered day 0 of gestation. The pregnant rats were then weighed and kept in cages until day 14 of gestation. Pregnant rats were divided into two groups: a control group (C); which was given normal saline, and a DEX group; which received 0.4mg/kg DEX (Catalogue \# D1159-500MG, SigmaAldrich Co., USA) as an intraperitoneal injection starting from the $14^{\text {th }}$ day of gestation $(\mathrm{dg})$ until the day of sacrifice (19 or $21 \mathrm{dg}$ ).

Laparotomy was carried out for the pregnant rats and uterine horns were obtained and placed on ice. Placentas were then acquired, cleaned and weighed, after which they were dissected for obtaining the basal zone (BZ) and labyrinth zone (LZ). Samples for PCR and western blotting were immersed in liquid nitrogen before storing at $-70^{\circ} \mathrm{C}$. Whole placentas were placed in $10 \%$ formalin for immune histochemistry.

\section{Real-time PCR}

TRIzol (Invitrogen USA catalogue \# 15596-018) was used for extracting total RNA as explained in our previous work. ${ }^{28,29}$ For each $0.1 \mathrm{~g}$ of sample, $1 \mathrm{ml}$ of TRIzol was added and the samples were homogenized. After that, Chloroform $(0.2 \mathrm{ml}$ to each $1 \mathrm{ml}$ TRIzol added) was added to each sample, after which the samples were centrifuged at $12,000 \mathrm{x}$ g for $10 \mathrm{~min}$. The aqueous phase was transferred to a new tube and ice-cold isopropanol was added and the mixture was incubated at room temperature for $10 \mathrm{~min}$. The mixture then was centrifuged at $12,000 \mathrm{x} g$ at $4^{\circ} \mathrm{C}$ for $10 \mathrm{~min}$. The supernatant was removed, and $1 \mathrm{ml}$ of $75 \%$ ethanol was added to the pellet. The samples were vortexed and centrifuged at $7500 \mathrm{x}$ g at $4^{\circ} \mathrm{C}$ for 5 min. The supernatant was discarded and the pellets were left to air dry for $15 \mathrm{~min}$. The pellets were then dissolved in $25 \mu$ l of Diethyl pyrocarbonate (DEPC) water at $55^{\circ} \mathrm{C}$ in a thermo-mixer for $10 \mathrm{~min}$.

Spectrophotometric measurements were used to determine the quantity of total RNA samples. The total RNA samples were diluted with DEPC water (1:500 dilution). The DEPC water was considered as blank and it was treated in the same way as the sample. Quartz cuvettes were used and the absorbance (A) was read at 260 and 280 $\mathrm{nm}$. The purity of sample preparation was indicated by calculating the ratio of A260/A280; only samples with A260/A280 ratios (1.72.0) were used for further studies. RNA integrity was checked by electrophoresis using $1 \%(\mathrm{w} / \mathrm{v})$ agarose gel and confirmed after visualization of the $28 \mathrm{~S}$ and $18 \mathrm{~S}$ rRNA bands under an ultraviolet (UV) transilluminator.

Before reverse transcription, the RNA samples $(0.5 \mu \mathrm{g} / \mu \mathrm{l})$ were DNase-treated then reverse transcribed as described previously. ${ }^{30}$ The RT-PCR reactions were carried out for the following genes: BAX (\#4331182 Rn 01480160_g1), JNK (Jkamp) (\#4351372 Rn 01473305_m1) and the internal control 18S (catalog \# 4319413E) using the TaqMan universal master mix (catalog \# 4369016), all purchased from Applied Biosystems.

\section{Western blotting and immune histochemistry}

Placentas were thawed, homogenized and the protein was estimated. The samples containing $40 \mu \mathrm{g}$ of protein and rainbow marker (14,300 - 220,000 Daltons; catalog \# RPN756E, GE Healthcare Ltd., UK) were loaded on gradient gels (SDS-PAGE 4-20\%; catalog \#0025244 Tris-HEPES-SDS, Precast Polyacrylamide Mini Gels). The run and protein transfer was performed as described previously. ${ }^{30}$ Proteins were transferred on PVDF membranes and then processed as follows; membranes were blocked with $10 \%$ non-fat dry milk in TBS-T (Tris-Buffer Saline-Tween 20: $20 \mathrm{mM}$ Tris, $137 \mathrm{mM}$ $\mathrm{NaCl}, \mathrm{pH} 7.6$ and $0.1 \% \mathrm{v} / \mathrm{v}$ Tween) for 1 hour at room temperature. Membranes were then rinsed and washed with TBS-T ( $20 \mathrm{ml} /$ wash) and then incubated overnight at $4^{\circ} \mathrm{C}$ with $3 \mathrm{ml}$ primary antibody diluted in 10\% non-fat dry milk in TBS-T (BAX (P-19): sc-526; (1:200) (JNK (FL): sc-571 (1:200). After incubation, the membranes were rinsed and washed with TBS-T $(20 \mathrm{ml} /$ wash $)$. The membranes were incubated with the appropriate secondary antibody diluted in $10 \%$ non-fat dry milk in TBS-T for 1.5 hours at room temperature. After washing, the membranes were covered with ECL plus (ECL Western Blotting Detection Reagents RPN 2106, GE Healthcare) according to the manufacturer's instructions and exposed to films. ${ }^{30}$ After obtaining the results for BAX and JNK, the membranes were treated with $\beta$-actin antibody (internal control; Millipore Corporation,

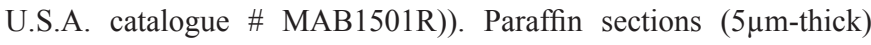
were deparaffinized using xylene and rehydrated in alcohol in declining concentrations of alcohol. The microwave oven was used for antigen retrieval using $0.01 \mathrm{M}$ citrate buffer. For determining the expression of the required genes, relative quantification was used. Hydrogen peroxide was quenched by using $3 \%$ hydrogen peroxide and to minimize background staining a $1 \%$ sodium borohydride and glycine of the concentration of $50 \mathrm{mM}$ were used. A blocking solution was then used as provided in the kit (cat \# Ref 858943 Histostain ${ }^{\circledR}$ plus Broad spectrum kit, Invitrogen, USA). The primary antibodies were diluted with PBS (primary antibodies were added to all slides 
except the -ve control to which PBS was added). The slides were kept in the humidified chamber for one hour at room temperature. Then, the slides were treated with the secondary antibody included in the kit (catalog \# REF858943 Histostain ${ }^{\circledR}$ plus Broad spectrum 1 kit, Invitrogen) for 30 minutes. Streptavidin was added (included in the kit; catalog \# REF858943 Histostain ${ }^{\circledR}$ plus Broad spectrum, Invitrogen) and kept for 30 minutes. The slides were rinsed with PBS twice, then ImmPACT-DAB chromogen-diaminobenzidine was added for two minutes then slides were visualized under the microscope, and color development was noted.

\section{Results}

\section{Dexamethasone induces IUGR and reduces placental growth in rats}

Compared to respective control groups, DEX treatment resulted in reduced fetal body weight on $21 \mathrm{dg}(\mathrm{p}<0.05$; Figure $1 \mathrm{~A})$. A significant reduction in total placental, basal zone, and labyrinth zone weights was seen on both 19 and $21 \mathrm{dg}(\mathrm{p}<0.05$; Figures $1 \mathrm{~B}, 1 \mathrm{C} \& 1 \mathrm{D})$. Based on these results, only $21 \mathrm{dg}$ placental samples were used in this study to investigate the expression of BAX and JNK.
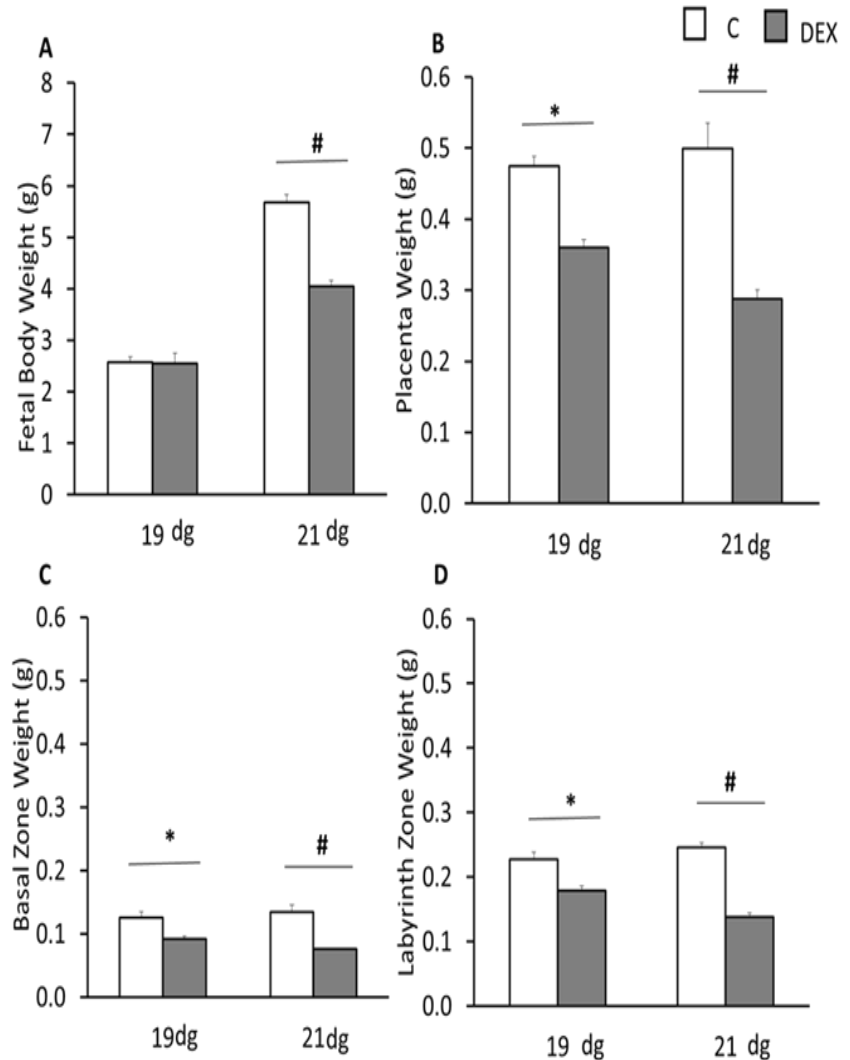

Figure I Effects of IUGR on fetal and placental weighs A, fetal body weight. $B$, placental weight. C, basal zone weight. D, labyrinth zone weight in control (C) and DEX (IUGR) groups at 19 and 21 days of gestation. ${ }^{p} p<0.05$ among $C$ or DEX groups; $\# p<0.05$ between $C$ and DEX groups. Data represent mean \pm S.E.M.

\section{DEX affects BAX and JNK gene expressions in rat placentas}

Both $B A X$ and $J N K$ mRNA expression decreased in the BZ in DEX placentas as compared to control placentas ( $<<0.05$; Figures $2-3 A$ ).
On the other hand, in the LZ, both genes increased in DEX placentas in contrast to control placentas ( $\mathrm{p}<0.05$; Figures $2 \mathrm{~A}-3 \mathrm{~A})$.
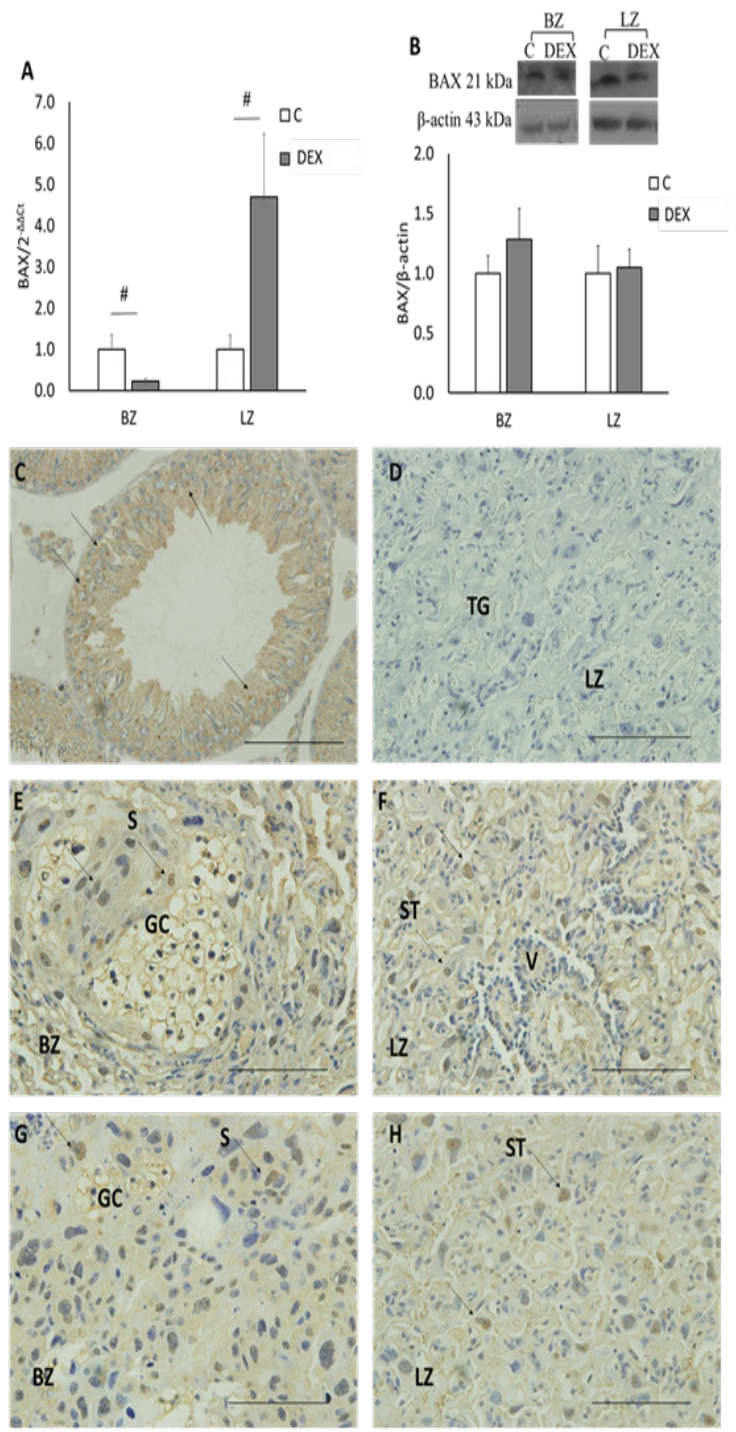

Figure 2 BAX gene, protein expression and localization in Basal (BZ) \& Labyrinth (LZ) Zones. Bands of apparent molecular weight of $\sim 2 \mathrm{I} \mathrm{kDa}$ and $\sim 43 \mathrm{kDa}$ were detected for BAX and $\beta$-actin, respectively. A) BAX gene expression in BZ \& LZ. B) BAX protein expression in BZ \& LZ. \#p $<0.05$ between $C$ and DEX groups. Data represent mean \pm S.E.M. C) The testis as a positive control for BAX using IHC staining. D) Negative control for $B A X$ using $I H C$ staining; cross section from rat placenta omitting the primary antibody. E \& F) localization of BAX in C placenta sections. G \& H) localization of BAX in DEX placenta sections. 40x magnification and a scale of $50 \mu \mathrm{m}$. ST: syncytiotrophoblast cells. BZ: basal zone. TG: trophoblast gaint cells. S: spongiotrophoblast cells. GC: glycogen cells. DC: deciduas cells; LZ: labyrinth zone.

\section{DEX did not affect BAX and JNK protein expression in rat placentas}

Both BAX and JNK were expressed in the BZ and LZ. A single band was detected for BAX protein $(\sim 21 \mathrm{kDa})$ while for JNK protein two bands were detected ( $\sim 54 \mathrm{kDa}$ and $\sim 46 \mathrm{kDa})$. In both zones the expression of BAX and JNK proteins showed no significant change among the groups (Figures 2B\&3B). 


\section{Localization of $\mathrm{BAX}$ in rat placenta}

In the BZ, the glycogen cells (GC) and spongiotrophoblast cells (S) were stained with BAX in both control and DEX placentas. While in the LZ, syncytiotrophoblast cells (ST) were stained with BAX in both control and DEX placentas (Figures $2 \mathrm{C}-\mathrm{H}$ ).

\section{Localization of JNK in rat placenta}

In the BZ, trophoblast gaint cells (TG) were stained with JNK in both control and DEX placentas. While in the LZ, ST were stained with JNK in both control and DEX placentas (Figures 3C-H).

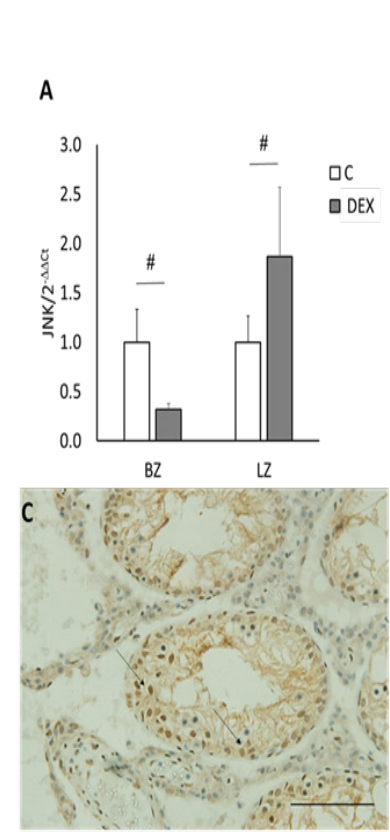

$$
\text { B } \quad \stackrel{\mathrm{BZ}}{\mathrm{C} D E X} \stackrel{\mathrm{LZ}}{\mathrm{C} \text { DEX }}
$$
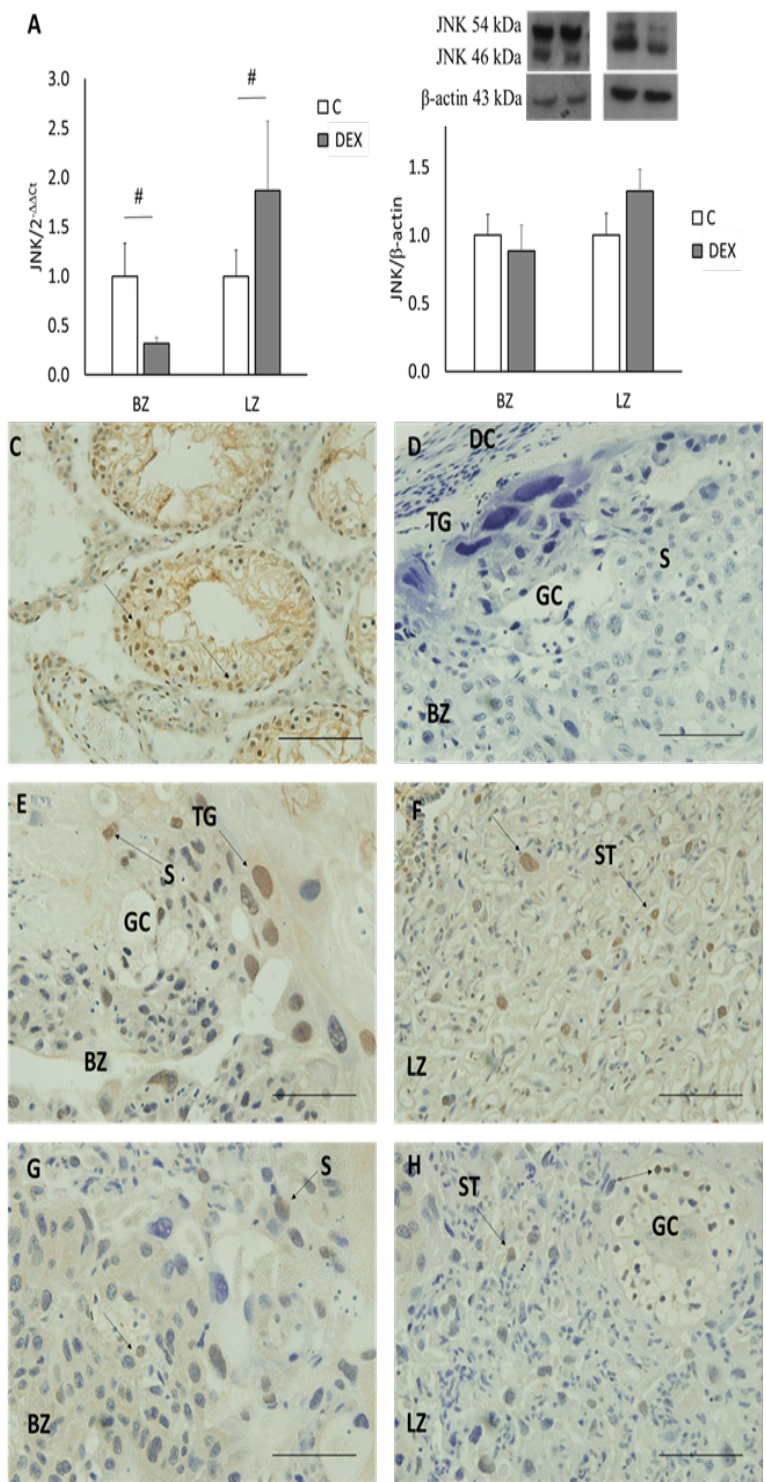

Figure 3 JNK gene, protein expression and localization in Basal (BZ) \& Labyrinth (LZ) Zones. Bands of apparent molecular weight of $\sim 54 \& \sim 46 \mathrm{kDa}$ (JNK) and $\sim 43 \mathrm{kDa}$ (actin) were detected.A) JNK gene expression in BZ \& LZ. B) JNK protein expression in BZ \& LZ. \#p<0.05 between C and DEX groups. Data represent mean \pm S.E.M. C) The testis as a positive control for JNK using IHC staining. D) Negative control for JNK using IHC staining; cross section from rat placenta omitting the primary antibody. E \& F) localization of JNK in C placenta sections. $G$ \& $H$ ) localization of JNK in DEX placenta sections. 40x magnification and a scale of $50 \mu \mathrm{m}$. ST, syncytiotrophoblast cells; BZ, basal zone; TG, trophoblast gaint cells; S, spongiotrophoblast cells; GC, glycogen cells; DC, deciduas cells; LZ, labyrinth zone.

\section{Statistical analysis}

All data are presented as mean \pm SEM. Data were tested for statistical significance by analysis of variance (ANOVA) between control and experimental samples and between the groups 19 and 21 followed by least significant difference (LSD) post hoc analysis using SPSS software as described earlier ${ }^{30}(p<0.05)$ value was considered the lowest level of significance.

\section{Discussion}

Since DEX is known to affect the normal placental development by increased levels of trophoblast apoptosis, ${ }^{11}$ it is therefore suggested that in DEX induced IUGR, apoptosis will be activated, leading to to a decrease in fetal body weight towards the end of gestation. In this study, JNK gene expression increased in the LZ suggesting an increase in cell apoptosis, as expected. This is also consistent with a previous research made by one of the members of our research, which showed up regulation of $\mathrm{p} 53$ expression in the LZ as JNK has been shown to stabilize and activate p53 being its substrate. ${ }^{30}$ The insignificant change in protein levels using western blotting maybe due to the presence of this protein in a single cell type in this zone. The decrease in the gene expression of JNK in basal zone indicates that other pro-apoptotic factors seem to play a role in decreasing the weight of this zone, suggesting that signaling molecules involved in apoptosis may be zone dependent.

Similarly, gene expression of BAX increased significantly in the LZ in DEX. This indicates that BAX contributes to apoptosis in the LZ, with the resultant decrease in placental weight in that zone. BAX gene expression in the BZ decreased, which may be due to that reduced BAX activation due to a change in phosphorylation by $\mathrm{JNK}^{27}$ which was shown to be decreased in the basal zone as mentioned above. The study done by our member using the same model showed that expression of p53 was increased in the LZ of DEX placentas, and it was observed that mobilization of $\mathrm{p} 53$ can induce apoptosis by upregulation of pro-apoptotic factors such as BAX, reviewed by ${ }^{31}$ This is consistent with our results which showed increased gene expression of BAX in the labyrinth zone. Apoptosis in the basal zone may be due to increased expression of other BCL2 family proteins, such as $\mathrm{BAK},{ }^{18,19}$ which can explain the decrease in weight in this zone. In our study, the level of BAX protein did not show significant difference between the control and experimental groups, this is most likely due to the analysis of all cell types in the LZ using western blotting, as previously mentioned. According to immune histochemistry results, only the ST cells stained positively for this protein.

\section{Conclusion}

In conclusion, our results indicate that BAX and JNK contribute to apoptosis in the labyrinth zone of IUGR placentas. The increased cell death in the BZ may be due to other pro-apoptotic factors of the BCL2 family members.

\section{Acknowledgments}

None.

\section{Conflicts of interest}

The authors declare there are no conflicts of interest.

\section{References}

1. Georgiades P, Ferguson Smith AC, Burton GJ. Comparative developmental anatomy of the murine and human definitive placentae. Placenta. 2002;23(1):3-19. 
2. Soares MJ, Chakraborty D, Karim Rumi MA, et al. Rat placentation: An experimental model for investigating the hemochorial maternal-fetal interface. Placenta. 2012;33(4):233-243.

3. Furukawa S, Kuroda Y, Sugiyama A. A Comparison of the Histological Structure of the Placenta in Experimental Animals. Journal Of Toxicologic Pathology. 2014;27(1):11-18.

4. Matt DW, Macdonald GJ. Placental steroid production by the basal and labyrinth zones during the latter third of gestation in the rat. Biology of Reproduction. 1985;32(4):969-977.

5. Baisden B, Sonne S, Joshi RM, et al. Antenatal Dexamethasone Treatment Leads to Changes in Gene Expression in a Murine Late Placenta. Placenta. 2007;28(10):1082-1090.

6. Unek G, Ozmen A, Kipmen Korgun D, et al. Immunolocalization of PCNA, Ki67, p27 and p57 in normal and dexamethasone-induced intrauterine growth restriction placental development in rat. Acta histochemica. 2012;114(1):31-40.

7. Waddell BJ, Hisheh S, Dharmarajan AM, et al. Apoptosis in Rat Placenta Is Zone-Dependent and Stimulated by Glucocorticoids. Biology of Reproduction. 2000;63(6):1913-1917.

8. Smith SC. Increased placental apoptosis in intrauterine growth restriction. American Journal of Obstetrics and Gynecology. 1997;177(6):13951401 .

9. Hewitt DP, Mark PJ, Waddell BJ. Glucocorticoids prevent the normal increase in placental vascular endothelial growth factor expression and placental vascularity during late pregnancy in the rat. Endocrinology. 2006;147(12):5568-5574.

10. Bauer MK, Harding JE, Bassett NS, et al. Fetal growth and placental function. Mol Cell Endocrinol. 1998;140(1-2):115-120.

11. Waddell BJ, Hisheh S, Dharmarajan AM, et al. Apoptosis in rat placenta is zone-dependent and stimulated by glucocorticoids. Biology of reproduction. 2000;63(6):1913-1917.

12. Roberts D, Dalziel S. Antenatal corticosteroids for accelerating fetal lung maturation for women at risk of preterm birth. Cochrane Database Of Systematic Reviews. 2006;(3).

13. Elmore S. Apoptosis: A Review of Programmed Cell Death. Toxicologic Pathology. 2007;35(4):495-516.

14. Danial NN, Korsmeyer SJ. Cell death: Critical control points. Cell. 2004;116(2):205-219.

15. Gross A, McDonnell JM, Korsmeyer SJ. BCL-2 family members and the mitochondria in apoptosis. Genes and Development. 1999;13(15):18991911.

16. Reed JC. Bcl-2 and the Regulation of Programmed Cell Death. The Journal of Cell Biology. 1994;124(1/2):1-6.

17. Kuwana T, Mackey MR, Perkins G, et al. Bid, Bax, and Lipids Cooperate to Form Supramolecular Openings in the Outer Mitochondrial Membrane. Cell. 2002;111(3):331-342.
18. Wei MC, Lindsten T, Mootha VK, et al. tBID, a membrane-targeted death ligand, oligomerizes BAK to release cytochrome c. Genes Dev. 2000;14(16):2060-2071.

19. Zong WX, Li C, Hatzivassiliou G, et al. Bax and Bak can localize to the endoplasmic reticulum to initiate apoptosis. J Cell Biol. 2003;162(1):5969.

20. Cui J, Zhang M, Zhang YQ, et al. JNK pathway: diseases and therapeutic potential. Acta Pharmacol Sin. 2007;28(5):601-608.

21. Davies C, Tournier C. Exploring the function of the JNK (c-Jun N-terminal kinase) signalling pathway in physiological and pathological processes to design novel therapeutic strategies. Biochem Soc Trans. 2012;40(1):8589.

22. Sui XB, Kong N, Ye L, et al. p38 and JNK MAPK pathways control the balance of apoptosis and autophagy in response to chemotherapeutic agents. Cancer Letters. 2014;344(2):174-179.

23. Dhanasekaran DN. JNK signaling in apoptosis. Oncogene. 2008;27(48):6245-6251.

24. Davies C, Tournier C. Exploring the function of the JNK (c-Jun N-terminal kinase) signalling pathway in physiological and pathological processes to design novel therapeutic strategies. Biochemical Society Transactions. 2012;40(1):85-89.

25. Saelens X, Festjens N, Vande Walle L, et al. Toxic proteins released from mitochondria in cell death. Oncogene. 2004;23(16):2861-2874.

26. Kim BJ, Ryu SW, Song BJ. JNK and p38 kinase-mediated phosphorylation of Bax leads to its activation and mitochondrial translocation and to apoptosis of human hepatoma HepG2 cells. J Biol Chem. 2006;281(30):21256-21265.

27. Tsuruta F, Sunayama J, Mori Y, et al. JNK promotes Bax translocation to mitochondria through phosphorylation of 14-3-3 proteins. EMBO J 2004;23(8):1889-1899.

28. Al-Bader MD, Jasem SA, Kilarkaje N. Carbenoxolone exposure during late gestation in rats alters placental expressions of p53 and estrogen receptors. Eur J Pharmacol. 2016;791:675-685.

29. Ghadhanfar E, Alsalem A, Al Kandari S, et al. The role of ACE2, angiotensin-(1-7) and Mas1 receptor axis in glucocorticoid-induced intrauterine growth restriction. Reprod Biol Endocrinol. 2017;15(1):97.

30. Alqaryyan M, Kilarkaje N, Mouihate A, et al. Dexamethasone-Induced Intrauterine Growth Restriction Is Associated With Altered Expressions of Metastasis Tumor Antigens and Cell Cycle Control Proteins in Rat Placentas. Reprod Sci. 2017;24(8):1164-1175.

31. Wu X, Deng Y. Bax and BH3-domain-only proteins in p53-mediated apoptosis. Front Biosci. 2002;7:151-156. 